\title{
To the space by laser light (II)
}

\author{
Victor V. Apollonov
}

A. M. Prokhorov General Physics Institute, Moscow, Russia; vapollo@kapella.gpi.ru

Received 25 May 2012; revised 23 June 2012; accepted 9 July 2012

\begin{abstract}
New approach to the problem of laser jet engine (LJE) creation is based on the use of shock waves (SW) resonance association mechanism, generated by the optical pulsating discharge (OPD). For the creation OPD is proposed to use powerful pulse-periodic (P-P) laser radiation with duration of pulses $\sim 100-150$ ns and high pulse repetition frequency $\sim 50-100 \mathrm{kHz}$. OPD is formed with the help of matrix of reflectors (MR). This is allowed: several times to increase the effectiveness of laser emission use for purposes of LJE creation, to avoid strong impact loads on the apparatus, to exclude the thermal action of laser plasma on the reflector, to decrease the laser emission screening by plasma, to remove the problem of the resonance oscillations appearance into the object moved with the help of laser. In our article the estimations of possible LJE thrust levels under the conditions of the proposed mechanism of reactive motion are given. Also, the new approaches to the creation of the high power high repetition rate laser systems for the "Impulsar" program are examined.
\end{abstract}

Keywords: Lightcraft; Impulsar; Launching of Objects by Laser; High Power Lasers; Optical Breakdown; Shock Waves

\section{INTRODUCTION}

LJE is the most promising rocket engine of a new class. In our article the discussion deals with the creation of automatic spacecraft engine, which will pass its trajectory under the action of directed toward it from the Earth's surface the sequence of high repetition rate short in time duration laser pulses.

The importance of this problem is caused by that circumstance that LJE is substantially more economical than traditional chemical-propellant engines. In the initial stage of flight as the working medium adapts atmospheric air, and beyond the limits of the atmosphere the insignificant onboard reserve of gas or easily sublimated substance. In this case specific expenditures for the launch of loads into space can be reduced to 5000 $10,000 \mathrm{rub} / \mathrm{kg}$, which is approximately 100 times less in the comparison with the contemporary scale of world prices. Especially, the possibility of maintaining the orbital parameters at the assigned level with the aid of the same laser system, intended for the starting, can be evaluated very highly.

In the present time the set of works on the study of LJE designing possibility is under very intensive realization. Within the framework project "Lightcraft" the intensive developments of such systems are conducted in USA, Germany and Japan. So, in November 2000 year American company "Lightcraft Technologies" successfully conducted model tests of rocket, which rose to the height $-70 \mathrm{~m}$ during $12.7 \mathrm{~s}$. under the action of exhaust jet, which appears under the effect of powerful laser emission. In the experiments the low-frequency $20 \mathrm{~Hz}$ $\mathrm{CO}_{2}$-laser with output power $10 \mathrm{~kW}$ was used, which in the present time is under upgrading to the level of the output power-100 kW. Mechanical impulse appeared due to ablation of special polymeric material from the concave surface of the reflector, located in the tail end of the rocket, where the laser beam pulses were accepted.

In the beginning of 1973 in the USSR under scientific guidance of Acad. A. M. Prokhorov the work on the study of possibility of LJE designing was conducted. The reflector, located in the tail of the rocket prototype, concentrated on the obtained radiation in air and ensured micro-burst that the reactive thrust was created. The successful results of different models of the reflectors tests, which were at the same time the laser light receivers, had been obtained. One should note that all experiments pointed out above were conducted with the use of electric discharge $\mathrm{CO}_{2}$-lasers with power up to $10 \mathrm{~kW}$, while for the injection into orbit of different highly and technologically effective equipments (global network connections, Internet, photo-monitoring of Earth surface, debris cleaning) the radiated power substantially higher is required thus, for example, for KA launching with the weight $1000 \mathrm{~kg}$ the laser with power not less than $10-15$ MW is necessary. This laser at present time can be the gas-dynamic laser (GDL) only, since only in this case the 
laser technology in the significant measure intersects the rocket technology, which in 50 years moved sufficiently well in the creation of super-power gas generators and it makes it possible to pose similar problems. Furthermore, the laser must work in P-P regime with the high frequency of the repetition of short pulses for the exception of the process of laser emission screening entering by the plasma, which appears with the engine work, and so for an increase in the work effectiveness [1]. In the opinion of specialists - classical missileman-LJE can find an effective use in creation of cheap single-stage means of nano- and micro-satellites launch with mass in the gap 5 - $50 \mathrm{~kg}$, which according to the opinion of experts tomorrow will compose the fundamental basis of commercial launch program. In the first stage of flight KA at the heights up to $30 \mathrm{~km}$ as the working medium in the engine the usage of atmospheric air is intended, and then to inject satellite into orbit the onboard reserve of the special fuel-ablating substance - in the quantities not exceeding $15 \%-20 \%$ of the KA started weight is needed.

The experience of powerful lasers creation is accumulated in the A. M. Prokhorov General Physics Institute of RAS, in the NPO "Energomash" after V. P. Glushko and other organizations of Russia. In OOO "Energomashtechnika" during last few years very successful experimental studies of P-P regime in the powerful and well developed continuous laser systems were conducted. This makes it possible to approach the experimental realization of super-power P-P laser source on the basis of gas-dynamic principle and LJE in the composition of light carrier with control system. The forthcoming complex of works must become major step toward the future starting of super-lightweight KA into low near Earth orbit. The realization of project will make it possible to create highly economical LJE of reusable starting for the removal of the payloads of wide designation into space. The key advantage of new approach is connected with the fact that the source of kinetic energy and payload are untied in the space and launching weight KA can be lowered to the payload weight only. An even greater K. E. Tsiolkovskii foretold, that the starting KA of future will be achieved with the aid of the electromagnetic waves directed from the external energy source, laser at that time was not known to the world at all. The great interest of scientists and specialists, as they confirmed few last symposium in this field of research, was connected with the successful solution in our country of the problem of powerful lasers creation with high repetition rate (50 $100 \mathrm{kHz})$ of pulses of short duration (150 - $250 \mathrm{~ns})$. This is what very recently wrote "the father of laser reactive motion" Arthur Kantrowits:

"Dear Victor, thanks for the pleasure given to read your articles from the last symposium on the motion with the aid of laser energy which are now accessible for the scientific community. I already thought above your works and I count that these remarkable ideas on the quasistationary wave, about the light-detonation waves, about the matrix of reflectors those introduced into your new examination are very important for the development of theory and technology of motion with the aid of the light. With great admiration I do control the development of the laser starting in the world and in Russia in particular. I hope that after your publications the significant activity in the field of laser engine creation and its applications will be stimulated. It would be very interestingly and further hear about the progress in this hot field of research. Thanks for your energetic contribution and be healthy.

A. Kantrowits".

High-frequency P-P mode of laser operation is developed and tested on the basis of powerful $\mathrm{CO}_{2}$-GDL, $\mathrm{Nd}$ YAG with lamp pumping and can be successfully used for other types of the powerful lasers, such as: HF/DF, Nd YAG with LD pumping, COIL and CO. The results of computer modeling for high repetition rate P-P lasers are presented in our review. The data of modeling are dependable on the characteristics of media, way of pumping, resonator geometry, and many other parameters of the laser under consideration [2].

1) COIL, P-P mode starts at frequencies $>20 \mathrm{kHz}$, depth of modulation $-100 \%$ starts at frequencies $>100$ $\mathrm{kHz}$, pulse duration $<250$ ns. Ratio: $\mathrm{P}$ peak./Paver. $=$ $100-1000$.

2) HF/DF, P-P mode starts at frequencies $>100 \mathrm{kHz}$, depth of modulation $-100 \%$ starts at frequencies $>250$ $\mathrm{kHz}$, pulse duration $<150$ ns. Ratio: $\mathrm{P}$ peak./Paver. $=$ $1000-10000$.

3) Nd YAG, P-P mode starts at frequencies $>4 \mathrm{kHz}$, depth of modulation $-100 \%$ starts at frequencies $>40$ $\mathrm{kHz}$, pulse duration $<250$ ns. Ratio: $\mathrm{P}$ peak./Paver. $=$ $100-1000$.

4) $\mathrm{CO}, \mathrm{P}-\mathrm{P}$ mode starts at frequencies $>10 \mathrm{kHz}$, depth of modulation $-100 \%$ starts at frequencies $>100 \mathrm{kHz}$, pulse duration $<250$ ns. Ratio: $\mathrm{P}$ peak./Paver. $=100-$ 1000.

At present time the project of the GDL with an output power of $20 \mathrm{MW}$ with the varied high-frequency structure of emission is located in the stage of active study in our country. Meaningful results on a notable increase in the effectiveness of laser energy use in the developed mode of emission, which made it possible according to our estimations to pass from tens of $\mathrm{kg}$ pay-load to a hundred, or even a thousand $\mathrm{kg}$ are obtained. So, one should note that the works in this region, on the basis of their large prospect for different applications, already begun in Germany, Japan, England, France, China, S. Korea, Brazil and a number of others countries. In this case, practically all participants of that works note the 
chosen position of GDL as the most promising system from the point of view of its scalability on the basis of the rocket technology to the level of several ten megawatts and other parameters, important for this kind of applications.

To the present time two directions, in which the possibility of applying the laser emission in the aerospace tasks is investigated: launching to the space orbit light $\mathrm{KA}$, reduction of aerodynamic drag of the flying bodies, which move in the atmosphere with the high speed were formed. In the problem of developing LJE the generated by P-P laser emission is focused by reflector near the rear end of KA and the periodically repetitive laser sparks are created. Sparks generate shock waves, which transfer the part of their mechanical pulse to the reflector, located near the tail of the rocket. As the characteristic of the previous years the gas-discharge laser systems had demonstrated the pulse repetition frequency, which was limited by the time of gas exchange in the discharge zone in the range of $100-300 \mathrm{~Hz}$. For achievement high average power-10 MW for this rate of operation it should be necessary to use laser pulses with the energy about 100 $\mathrm{kJ}$. With the reduced air pressure, for example for the height more than $15 \mathrm{~km}$, long living plasma sphere, created by each pulse, occupies practically entire volume of reflector, which leads to the screening of the subsequent pulses into the course $\sim$ of $10 \mathrm{~ms}$. Technical difficulty of that method of energy extraction for the laser is also a very strong impact loads with such a high energy of pulses. The use of high energy pulses with the small repetition frequency and, consequently, with the very high peak power is limited also by optical breakdown as on the route, so on the surface of reflector. The method proposed by us is the way of overcoming all that difficulties on the indicated basis: usage of laser emission with short duration of pulse and high pulse repetition frequency and mechanism of generated by OPD shock waves resonant association. Under that conditions of LJE energy of laser pulse with the short duration (100 ns.) effectively (95\%) can be absorbed and converted ( 30\%) into shock waves. In addition to this it is shown that the specific thrust can be increased several times due to the artificial transformation of radial component of shock waves into the longitudinal one. There are many other advantages of high repetition rate P-P lasers should be mentioned here as well [3]. They are:

1) Much higher efficiency of energy extraction from a media and conversion into laser light, which is important for high aperture lasers scale up;

2) Plasma screening effect decreasing due to the decreasing of laser pulses duration and increasing of number of pulses up to a few tens of $\mathrm{kHz}$;

3) Decreasing of laser beam thermal defocusing due to the optimal temporal structure of laser radiation;

4) Localization of energy deposition in space and time and elimination of stressed state of solids under laser action due to replacement of melting by ablation;

5) Possibility of unrolled 3 d geometry of breakdowns in space due to a very high repetition rate generation of laser pulses, creation of optical, acoustical and electromagnetic fields far away of laser source;

6) Much longer distances of optical breakdown conditions for the same optical systems.

\section{SPARK PARAMETERS FOR LJE}

Laser emission is focused by the reflector, which can have a form of hemisphere or parabolic. In Figure 1(a) the significant dimensions of reflector, focusing one spark, and also plasma zone, created by spark are shown. Distance $F_{f}$ must be little between the focal point and the reflector $F_{f} / R_{d}<0.2$, which follows from the condition of achieving the high value of the specific impulse of thrust. It follows in avoiding of the optical breakdown on the reflector and of the conditions of transport that the beam must have large diameter on the reflector. If the intensity of emission exceeds the threshold of optical breakdown, then plasma front is extended towards the ray, occurs heating and the ionization of air as a result of absorbing the laser emission. Since intensity in the sharply focused ray rapidly decreases (shape factor), then already at the small distance from the focus occurs the disruption of the

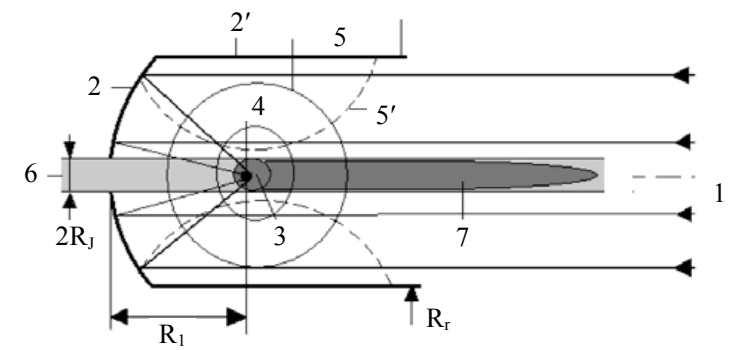

(a)

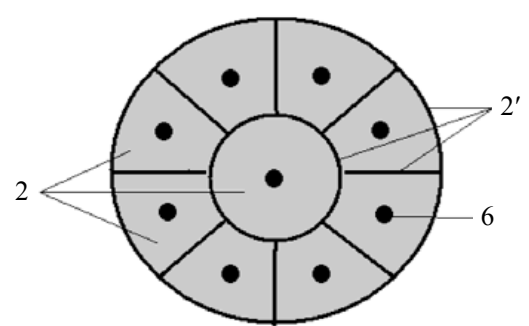

(b)

Figure 1. Scheme of reflector (a) and possible structure of matrix of reflectors (b) for LJE. 1: high rep. rate P-P laser radiation; 2: back side of reflector-zone of laser pulses interaction; 2': side wall of reflector; 3: cavern; 4: OPD; 5: shock wave; 5': reflected shock wave; 6: gas stream; 7: plasma stream. 
light-detonation regime of the propagation of plasma front. Further, emission is absorbed for a while in the decay plasma. It follows from the calculations carried out by us that for LJE the greatest interest represents the pulses with the duration $\sim$ of $0.2 \mathrm{~s}$. Maximum energy of laser pulses is limited by the condition for the achievement of a high efficiency in the use of laser emission for the thrust application. In the free gas space, the pulse transferred by shock wave is different from zero only at the small distances from the burst center. The same data were observed also in the early experiments. For the reflector of parabolic form the specific impulse is maximum $\sim 550 \mathrm{~N} \cdot \mathrm{s} / \mathrm{J}$ at the distance $\mathrm{R}_{1}$, equal with the short duration of the pulse of $\sim 100$ - $200 \mathrm{ns,} \mathrm{approximately,} \mathrm{the}$ tenth of a dynamic radius, or by another words, the distance, where the value of pulse diminishes is approximately three times bigger. With the increase $R_{1} / R_{d}$ from $\sim 0.1$ to 0.33 the value $\mathrm{J}_{1}$ it rapidly decreases $\sim$ by 550 $200 \mathrm{~N} \cdot \mathrm{s} / \mathrm{J}$. By the case LJE the possibility of using the low values $R_{1} / R_{d}$ it is limited by the formation of the long-life plasma, whose characteristic radius is compared with the dimensions of reflector. At the last stages of the thermal expansion of spark is formed plasma zone with the low density and the high temperature $\sim 8000 \mathrm{k}^{0}$ the ionized gas. The contact boundary of hot region stops with the pressure balance in the plasma and the surrounding gas. In the approximation of the spherical form of spark a radius of plasma region at the pullout moment of pressure it is possible to obtain the estimation of a radius of plasma formation for entire operating range of the pressures $0.1-1 \mathrm{~atm}$. The ratio of a radius of plasma zone to a dynamic radius in this case composes the value $0.15-0.25$. This relation does not depend on energy and the gas pressure. Moreover its value is located in the same range, where is attained the maximum the value of the specific recoil momentum, created by shock wave. In the reflector, which has the form of hemisphere, the maximum the specific impulse of the thrust has reached at $\mathrm{R}_{1} / \mathrm{R}_{\mathrm{d}} \approx 1$. Let us give the values of a radius of plasma formation and time of its formation for energy $10^{5} \mathrm{~J}$ and the pressures of atmosphere 1 and $0.1 \mathrm{~atm}$. The parameters correspond: peak pulse power $-2 \times 10^{7} \mathrm{~W}$ and to the pulse repetition frequency $-100 \mathrm{~Hz}$, and also to start and to the end of the stage of acceleration KA in the regime LJE. With pressure $1 \mathrm{~atm}$. The size of plasma sphere will be $25 \mathrm{~cm}$. With the time of formation on the order of 1 $\mathrm{ms}$, while at a pressure of the atmosphere $-0.1 \mathrm{~atm}$. The size of plasma sphere will comprise already $50 \mathrm{~cm}$ with the time of formation $-2 \mathrm{~ms}$. Cooling laser plasma occurs due to the turbulent mixing with the cold surrounding gas. The characteristic time of this process more than to the order exceeds the time of its formation. Here the spark with spherical form has been examined. On the matter itself the spark in LJE has a form of cone with the large apex angle. This even more worsens situation. With the specified conditions at the last stage of the expansion of spark can be formed the cumulative jet, in which the gas moves in the direction to the reflector. Plasma ceases to be singly connected, taking the form, similar to the torus. Thus, the use of a regime with the maximum pulse, presented in the early work of many authors, it will be accompanied by the contact of plasma with the surface of reflector, which, undoubtedly, will lead to its destruction.

\section{MECHANISM OF SHOCK WAVES RESONANCE ASSOCIATION}

The effect of resonance association separate shock waves into the low-frequency quasi-stationary wave (QSW) in the general case consists in following. In continuous medium consecutively are created periodic disturbances-shock waves, whose initial velocity is more than the speed of sound. If the speed of the displacement of the field of pulsations is lower than the speed of sound in the medium, then shock waves are united and are created BY SQ. Depending on the time-spatial structure of pulsations, the mechanism is manifested in the form the effects, basic property of which is the large length of high-pressure area. The mechanism of shock waves association does not contain limitations to the type of medium and source of pulsations, to its energy. Depending on the structure of pulsations, QSW can have various forms.

The realization of mechanism $\mathrm{kV}$ makes it possible to remove the problems examined above: the screening of emission and the thermal action of laser plasma in LJE. Furthermore, QSW makes it possible to substantially increase the effectiveness of the use of laser emission in the calculation of an increase in the specific thrusts by the unit of power. Let us determine requirements for energy and pulse repetition frequency of P-P of the laser emission, which satisfy solution of this problem. In this case has the sense to examine two methods, based on the use "spherical" and "flat" OPD. In both cases is formed flat short circuit the calculation of the use of both the geometry OPD and the geometry of reflector, and also the organization of the process of the introduction of energy of laser emission into the engine.

\section{LJE ON THE BASIS OF SHOCK WAVES RESONANCE ASSOCIATION FOR THE CASE OF MR}

In the general case MR is represented in the Figure 1(b). The problem of developing LJE on the basis of shock waves merging and P-P of laser emission with the high (to $100 \mathrm{kHz}$ ) pulse repetition frequency, and also flight path control aid LJE it is decided with the use MR. The schematic of engine is collection from $\mathrm{N}$ mono- 
reflectors. To MR brings by P-P the emission with the energy of the pulses $q$ with the average power $\mathrm{W}_{\mathrm{C}}$. In the general case the elements MR are identical. MR creates matrix OPD, each of which is stabilized by the air jet of the incident flow. A quantity of matrix elements in the general case determines the high-speed regime of gas jet. In our case a quantity of elements in the matrix was $\mathrm{N}=$ 8 .

In our calculation of power of laser emission was taken as the equal of $20 \mathrm{MW}$, the pulse repetition rate$100 \mathrm{kHz}$, energy of each pulse in this case was equal 200 J. Calculations showed that for the accepted for the examination parameters in the case of normal atmospheric pressure the complete engine thrust will be equal to 100 thousand $\mathrm{N}$, and at a pressure of the order of the tenth atmosphere its value will fall to the value-36 thousand N.

The optimum gas flow speed for both cases proved to be equal to $2.5 \mathrm{~km} / \mathrm{s}$ and $5.4 \mathrm{~km} / \mathrm{s}$ respectively. One should also note that the flight control is possible with the aid of the laser engine for the calculation of a change in the thrust in the equivalent components MR. However, an increase in the number of elements MR is accompanied by a significant increase in aerodynamic drag on the air ducts and the selected number of elements is close to the optimum.

In the experiments the model of rocket with the laser engine was the cylinder with diameter $\sim 8 \mathrm{~cm}$, with the length $\sim 26 \mathrm{~cm}$ and with the weight $1.1 \mathrm{~kg}$, suspended to four thin wires with total length of $1.1 \mathrm{~m}$ (Figure 2). Construction allowed displacement only in the axial direction. On the chamber end is fixed the reflectorchange cylindrical cap. Laser emission was introduced into the camera through the lens with a focal length of 17 $\mathrm{cm}$. The jet of argon was formed with the expiration from the high-pressure chamber through the opening with a diameter of $\sim 3$ or $4 \mathrm{~mm}$. The speed was regulated by the pressure of argon, which was given into the camera on the flexible hose. The force, created by jet and shock waves, was transferred with the aid of the thin $(\sim 0.2 \mathrm{~mm})$ molybdenum wire to the load, which stands on the strain scale weights.

OPD burnt in the flow, which was formed with the outflow of gas from the camera through the opening in the reflector. OPD was created by P-P laser emission$\mathrm{f}=50 \mathrm{kHz}$ and $100 \mathrm{kHz}$, in this case the average radiated power comprised $\mathrm{P} \approx 1200 \mathrm{~W}$. Thrusts with $\mathrm{f}=50 \mathrm{kHz}$, The $\mathrm{V}=300 \mathrm{~m} / \mathrm{c}$ it proved to be equal $-40 \mathrm{~g}$, and with The $\mathrm{V}=400 \mathrm{~m} / \mathrm{c}-69 \mathrm{~g}$. The value of the thrust coupling coefficient has proved to be equal-1.06 N/kW. Experiment confirmed that P-P laser emission creates stationary thrust with the very high coupling coefficient-1000 N/MW. A difference between the experimental results and theoretical estimations, whose value $>4500 \mathrm{~N} / \mathrm{MW}$, is connected with the need for further optimization of the process of the introduction of energy of laser into the plasma OPD.

\section{RESONANCE PROPERTIES OF SYSTEM "LASER-KA"}

By important parameter in the estimation of the resonance properties of system "Laser-KA" the ratio of the duration of laser pulse to the transit time of sound throughout the entire length of rocket appears. In the practical sense there is greatest interest in the case, when uniform load under the action of laser pulse is created throughout the entire length of rocket. If pulse has very short duration, then acceleration is non-stationary, the length of that excited in KA waves are much less than the length of rocket. The case, when the pulse duration and the transit time of acoustic wave are compared, corresponds to the resonant step-up of waves.

Let us make the resonance properties of system for the laboratory experiences in the case of KA with the mass$20 \mathrm{~kg}$, the length of the rocket of equal $-200 \mathrm{~cm}$, acceleration $-100 \mathrm{~m} / \mathrm{s}^{2}$. Average power of P-P radiation-4 $\mathrm{MW}$, the energy of pulses generated with repetition rate $-200 \mathrm{~Hz}$ is equal $-20 \mathrm{~kJ}$. Calculations show that for small pulse repetition rate of $\mathrm{P}-\mathrm{P}$ laser radiation the

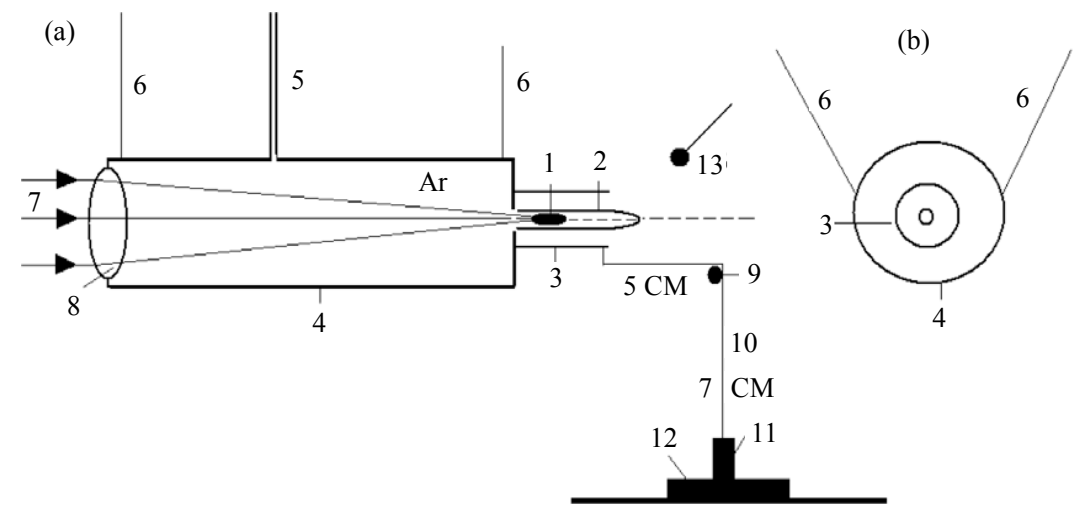

Figure 2. Scheme of experimental setup. 
acceleration mode is accompanied by the extremely strong dynamic structural loads KA. They have resonance nature, since the wavelength of compression is compared with the length KA. Furthermore, with an increase in the length KA about $4 \mathrm{~m}$ and the repetition frequency of the laser pulses up to $-1 \mathrm{kHz}$ the natural frequency of oscillation of rocket is equal to pulse repetition rate of laser and we obtain the fulfillment of conditions of resonance. This is very dangerous, since resonance loads can exceed the safety factor of rocket and it will be destroyed in the flight.

Thus, estimations show that the thermal contact of plasma with the reflector and strong dynamic loads are inevitable with the low pulse repetition frequency. Situation is aggravated by the excitation of resonance oscillations in the KA. These difficulties are surmounted only with the use of a method, based on the association of shock waves [4]. Calculations and experiment confirmed possibility of significant stationary thrusts designing with the use P-P of emission with high pulse repetition frequency.

\section{CONCLUSIONS}

In conclusion of the material presented, one should say that as a result of the conducted investigations LJE is possible the passage to the solution of the following completely interesting problems:

1) Creation of the interceptor of manmade space debris and other dangerous space objects, such as asteroids, comets, meteorites and so on;

2) Launch of micro-objects with the super-high acceleration to the space;

3) Realization of orbital scale conducting channels for energy delivery from space to the ground and vice-versa.

\section{REFERENCES}

[1] Apollonov, V.V., Vagin, Yu.S., Egorov, A.B., Kiyko, V.V. and Kislov, V.I. (2001) Patent RF No. 2175159.

[2] Apollonov, V.V. (2005) High power lasers for "lightcraft" applications. Proceedings of SPIE, 5958, 315-322.

[3] Apollonov, V.V., Vagin, Yu.S., Egorov, A.B., Kiyko, V.V. and Kislov, V.I. (2003) High-frequency pulse-periodic mode for high-power laser. AIP Conference Proceedings, 702, 357-366.

[4] Apollonov, V.V. (2010) Vpered k Tciolkovskomu. 